\title{
Effects of acupuncture and electroacupuncture on estradiol-induced inflammation and oxidative stress in health rodents ${ }^{1}$
}

\author{
Elba Lucia Wanderley Santos', Bruno Hállan Meneses Dias"I, Ana Carolina Rodrigues de AndradeII, Angélica Maria Holanda \\ Pascoal $^{\text {II, Francisco Eugênio de Vasconcelos FilhoII, Francisco das Chagas Medeiros }}{ }^{\text {III }}$, Sergio Botelho Guimarães ${ }^{\text {IV }}$ \\ IFellow Master degree, Postgraduate Program in Surgery, Federal University of Ceara (UFC), Brazil. Technical procedures, acquisition and interpretation \\ of data.

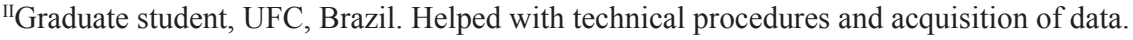 \\ IIIPhD, Associate Professor, Department of Maternal and Child Health, UFC, Brazil. Analysis of data, critical revision. \\ ${ }^{\text {IV }} \mathrm{PhD}$, Associate Professor, Department of Surgery, Head, LABCEX, UFC, Brazil. Conception, design, intellectual and scientific content of the study; \\ critical revision, final approval of manuscript.
}

\begin{abstract}
PURPOSE: To investigate the effects of classical acupuncture (Ac) and electroacupuncture (EAc) on estradiol-induced inflammation and oxidative stress in health rodents.

METHODS: Twenty-four eight-week old female rats were treated with estradiol valerate (EV) $4.0 \mathrm{mg}$ i.m. single dose and randomly assigned to four groups (n=6): G1(control), G2 (Ac), G3 (EAc 2 Hz) and G4 (EAc $100 \mathrm{~Hz}$ ). After 60 days all rats were anesthetized with chloral hydrate $10 \%(0.1 \mathrm{ml} / 30 \mathrm{~g}$ weight of the animal) and submitted to Ac/EAc for twenty minutes. The procedures were repeated on days three, five, seven and nine of the study. The equivalent of the human right ST-36 (Zusanli) and SP-6 (Sanyinjiao) acupoints were chosen for needling and electrical stimulation. On the 10th day of the experiment, all rats were anesthetized for collection of blood and tissues (ovaries) samples for biochemical analysis and histological examination.
\end{abstract}

RESULTS: Glutathione (GSH) and malonaldehyde (MDA) concentrations increased significantly in all groups (plasma and ovary) while myeloperoxidase (MPO) activity decreased significantly in all groups compared with control group (G1).

CONCLUSIONS: Both classical acupuncture and electroacupuncture decrease systemic and local oxidative stress and ovary inflammation in healthy rats exposed to estrogenic stimulation. EAc enhances lipid peroxidation at systemic and local levels in female rats exposed to estrogenic stimulation.

Key words: Acupuncture. Electroacupuncture. Lipid Peroxidation. Oxidative Stress. Rats. 


\section{Introduction}

Reactive oxygen species (ROS) are generated in all aerobic cells and include superoxide radical $\left(\mathrm{O}_{2}\right)$, hydrogen peroxide $\left(\mathrm{H}_{2} \mathrm{O}\right)$ and hydroxyl radical $\left(\mathrm{OH}_{2}\right)^{1}$. Mitochondria are not only responsible for energy (ATP) production, but also are a major source of reactive oxygen species $(\mathrm{ROS})^{2}$. Under physiological circumstances, there is a balance between antioxidants and oxidants produced by aerobic cellular systems. Because aerobic cells possess antioxidant systems to trap and/or inactivate such oxygen intermediates, oxidative stress is the result of an imbalance between the production of ROS and their inactivation by the protective systems ${ }^{1}$.

There are now numerous reports showing that estrogen and estrogen-like compounds are effective in protecting against a wide variety of insults in numerous different cell types ${ }^{3}$. Estrogens effectively prevent pro-oxidant stress in the mitochondria ${ }^{4,5}$. Tugay et al..$^{6}$ studied the effects of dexamethasone on oxidant/antioxidant status in kidneys of rats administered mercuric chloride and concluded that dexamethasone injection may partially protect the rodent kidneys against oxidative reactions by preventing the increase in xanthine oxidase activity. Estrogen has been shown to exert antioxidant properties in rodent hearts exposed to ironstimulated lipid peroxidation ${ }^{7}$. In aging liver oxidative stress increases due to the decrease in antioxidant bio-molecules such as estrogens which can be modified by hormonal replacement therapy. Long-time estrogen supplementation prevent the increase in lipid peroxidation induced by aging 8 .

On the other hand, adverse effects of steroids have been demonstrated. Dexamethasone is known to induce free radical toxicity in humans and animals ${ }^{9-11}$. Increased ROS generation resulting in liver oxidative stress has been demonstrated in sea bass exposed to $17 \beta$ estradiol $^{12}$. Recent experimental studies have demonstrated that chronic estradiol exposure induces oxidative stress in the hypothalamus of rats ${ }^{13}$. Long-acting compounds such as estradiol valerate (EV) may develop polycystic ovary syndrome (PCOS) in rodents with a single intramuscular (i.m.) injection ${ }^{14,15}$.

Whereas steroids may induce oxidative stress and that the use of acupuncture (AC) or electroacupuncture (EAc) can attenuate the oxidative stress in different organs and tissues such as liver and kidneys ${ }^{16}$ and random skin flaps ${ }^{17}$ in anesthetized rats, this study aimed to investigate the possible protective effects of Ac and EAc stimulation in young female rats exposed to a single high dose of EV.

\section{Methods}

Approval for experimental use of laboratory animals was obtained from the local Ethics Committee on Animal Use (CEUA, former CEPA) (Protocol\#107/09, December 2009). All surgical procedures and animal handling were conducted in accordance with the Brazilian Federal Law No. 11794 of October 8, 2008 (http://www.planalto.gov.br/ccivil_03/_Ato2007-2010/2008/Lei/ L11794.htm) and Decree No. 6689 of July 15, 2009 , available at http://www.planalto.gov.br/ccivil_03/_Ato2007-2010/2009/ Decreto/ D6899.htm. The study was designed so as to minimize the number of animals required for the experiments.

\section{Animal preparation}

Twenty-four female Wistar rats weighing 150-220g, provided by the Faculty of Medicine Small Animals Breeding Facility (Federal University of Ceara) were kept under controlled environmental conditions $\left(24^{\circ} \mathrm{C}\right.$ relative humidity $40 \%-60 \%$, 12-hour alternate light-dark cycles, food and water ad libitum). The equivalent of the human right ST-36 (Zusanli) and SP-6 (Sanyinjiao) acupoints were chosen for needling and electrical stimulation. The acupoint nomenclature used follows WHO nomenclature ${ }^{18}$. ST-36 acupoint is located $5 \mathrm{~mm}$ below the head of the fibula under the knee joint, and $2 \mathrm{~mm}$ lateral to the anterior tubercle of the tibia. SP-6 acupoint is located $5 \mathrm{~mm}$ above the medial malleolus, about an imaginary line connecting this point to another point in the depression below the medial condyle of the tibia $^{19}$

\section{Materials}

Needles (sterilized stainless steel, $0.25 \mathrm{~mm}$ in diameter, $1.5 \mathrm{~cm}$ long) where purchased from Xu Li Comércio, Importação e Exportação Ltda, São Caetano do Sul - SP, Brazil. EL-608 portable electro stimulator was purchased from NKL Produtos Eletrônicos Ltda, Brusque, Santa Catarina, Brazil.

\section{Experimental groups}

Animals were anesthetized intraperitoneally with a fresh-prepared solution of chloral hydrate $10 \%(0.1 \mathrm{ml} / 30 \mathrm{~g}$ body weight). Twenty-four rats were randomly assigned to 4 equal groups $(n=6)$ as follows: 
Group 1: (Control)

Group 2: Acupuncture (Ac)

Group 3: Electroacupuncture $2 \mathrm{~Hz}$ (EAc $2 \mathrm{~Hz}$ )

Group 4: Electroacupuncture $100 \mathrm{~Hz}($ EAc $100 \mathrm{~Hz})$

After one week of acclimatization, twenty-four 8-weekold rats received an i.m. injection of EV $4.0 \mathrm{mg}$ (Sigma-Aldrich Laboratory Ltda, Brazil), in $0.2 \mathrm{ml}$ of olive oil, in the quadriceps muscle. Sixty days later Groups 2, 3, and 4 rats were anesthetized as described and treated as follows:

Group 2 - After routine skin disinfection with 75\% ethanol sterilized disposable stainless steel needles $(0.25 \mathrm{~mm} \times$ $15 \mathrm{~mm}$ ) were inserted perpendicularly as deep as 2-3 $\mathrm{mm}$ at right ST-36 and SP- 6 acupoints, bilaterally. Needles were kept in place for 20 minutes.

Groups 3/4 - After needle insertion, as described, electrodes were connected to all needles and to the NKL electrostimulator; pulsed square waves, Burst type $1 \mathrm{~mA}, 2 \mathrm{~Hz}$ (Group 3) or $100 \mathrm{~Hz}$ (Group 4), with duration of two seconds and pause for two seconds were applied for 20 minutes. Ac / EAc applications were repeated every other day, at the same time, for a total of five applications, held on one, three, five, seven and nine days of the experiment. Group 1 rats served as positive control and did not receive any additional treatment. On the 10th day of the experiment, all rats were anesthetized as described. A midline laparotomy was performed for blood $(3.0 \mathrm{ml})$ and both ovaries were harvested for biochemical analysis and histological examination.

\section{Biochemical determinations}

Parameters determined included myeloperoxidase (MPO) malondialdehyde (MDA) and glutathione (GSH) concentrations. Tissue samples were snap-frozen in liquid nitrogen and stored in glass tubes at $-70^{\circ}$ until subsequent preparation and analysis of liver and kidney homogenates. Lipid peroxidation was assayed by measuring malondialdehyde as TBA-reactive substances ${ }^{20}$. In brief, $\mathrm{H}_{3} \mathrm{PO}_{4}(1 \%, 3 \mathrm{~mL})$ and aqueous TBA solution $(0.6 \%$, $3 \mathrm{~mL})$ were added to the $10 \%$ homogenate $(0.5 \mathrm{~mL})$. The assay medium was shaken and heated on a boiling-water bath for 45 min. After cooling, $4 \mathrm{~mL}$ of $n$-butanol was added and the mixture shaken. After separation of the $n$-butanol layer by centrifugation at $1200 \mathrm{~g}$ for $15 \mathrm{~min}$, its optical density was determined in a spectrophotometer (Beckman DU 640 B; Beckman Instruments, now Beckman Coulter, Inc., Fullerton, CA, USA) with 535 and $520 \mathrm{~nm}$ as absorption wavelengths, respectively. The difference between the results of the two optical density determinations was taken as the TBA value and the amount of malondialdehyde
(MDA) in the testis was calculated, comparing with MDA standards and expressed as micromoles of MDA per gram of wet tissue. GSH levels were estimated by the method of Sedlak and Lindsay ${ }^{21}$ which is based on the reaction between thiol groups and 5-5-dithiobis-(2-nitrobenzoic acid) to produce a compound that absorbs light at $412 \mathrm{~nm}$. The amount of GSH was determined from a standard curve simultaneously obtained under the same conditions with various concentrations of GSH.

\section{MPO activity}

In brief, after collection, the sample was placed in an Eppendorf tube with cold buffer $(0.1 \mathrm{M} \mathrm{NaCl}, 0.02 \mathrm{M} \mathrm{NaPO}$, NaEDTA 0.015 M, pH 4.7). The tissue was weighed and then homogenized in a Polytron PT 3.100 to $13.000 \mathrm{rpm}$. Hypotonic lysis was carried out in the pellet using $2 \% \mathrm{NaCl}$ solution; after $30 \mathrm{~s}, \mathrm{NaCl} 1.6 \%$ and glucose $5 \%$ were added. After centrifugation, the pellet was suspended and homogenized in $0.05 \mathrm{M} \mathrm{NaPO}_{4}$ buffer ( $\mathrm{pH} \mathrm{5.4)} \mathrm{containing} 0.5 \%$ hexadecyltrimethylammonium bromide. After centrifugation at 13,000 rpm, $5 \mathrm{~mL}$ of supernatant diluted in $45 \mathrm{~mL}$ of 0.08 was used to measure MPO activity in a 96-well plate. MPO activity in the supernatant was measured using tetramethylbenzidine (TMB) and $1.6 \mathrm{mM} \mathrm{H} 2 \mathrm{O}(0.5 \mathrm{mM})$ and read on the 96-well-plate reader at $450 \mathrm{~nm}$. The final concentration of MPO was obtained by comparing the absorbance value with a standard scale of MPO, prepared in advance. The concentrations of MPO were expressed as units/mg of tissue.

\section{Ovary preparation}

Upon removal of the ovaries, they were cleaned of adherent connective tissue and washed in saline. The right ovary was saved for biochemical analyses. The left ovary was fixed in $4 \%$ formaldehyde buffer for 24 hours, dehydrated and imbedded in paraffin. Next, the left ovary was partially longitudinally sectioned $(4 \mu \mathrm{m})$, mounted on the glass slide and stained with hematoxylin and eosin (HE).

\section{Data analysis}

Graphpad Prism 5.0 (GraphPad Software, San Diego California USA, www.graphpad.com) was used for graphics design and statistical analysis. All results were expressed as mean \pm SD. All data were tested for distribution (Kolmorogov-Smirnov test with Dallal-Wilkinson-Lilliefor P value). One-way ANOVA (Dunnett's multiple comparison test) was performed to determine differences 
among groups in MDA and GSH concentrations in blood and ovary tissues. A probability value of $\mathrm{p}<0.05$ was considered to indicate statistical significance.

\section{Results}

\section{Effects of VE on ovary structure}

No histological findings compatible with PCOS was identified in rats treated with VE (Figure 1).

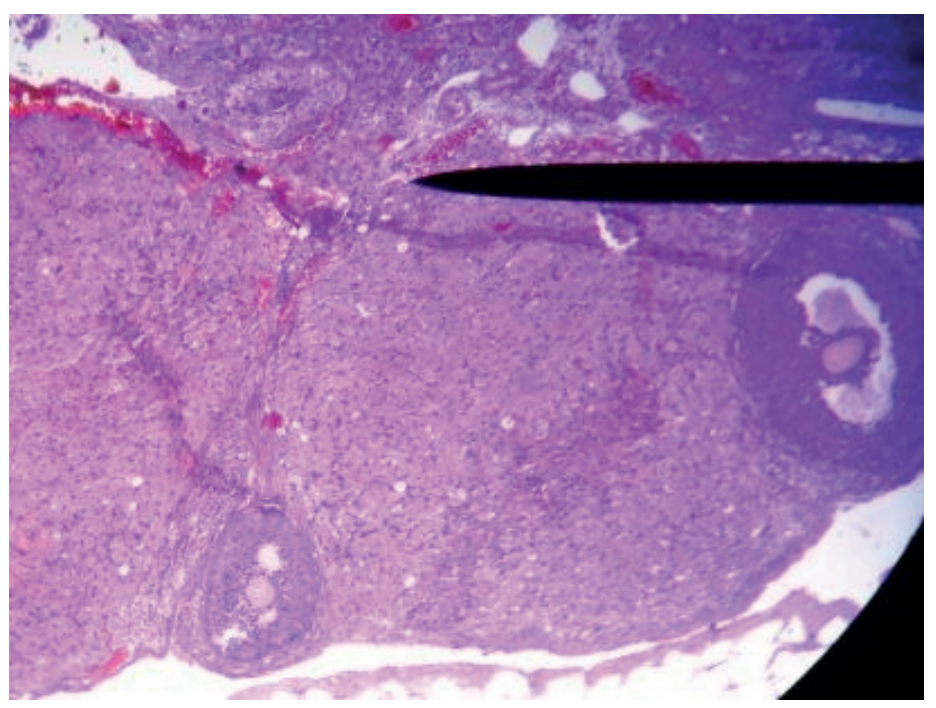

FIGURE 1 - Photomicrograph of left ovary from control group (G1) exhibiting typical normal appearance with follicles and corpora lutea in different stages of development and regression (HE, x40).

\section{GSH assay}

Plasma GSH concentrations increased significantly in all groups compared with control (Figure 2).

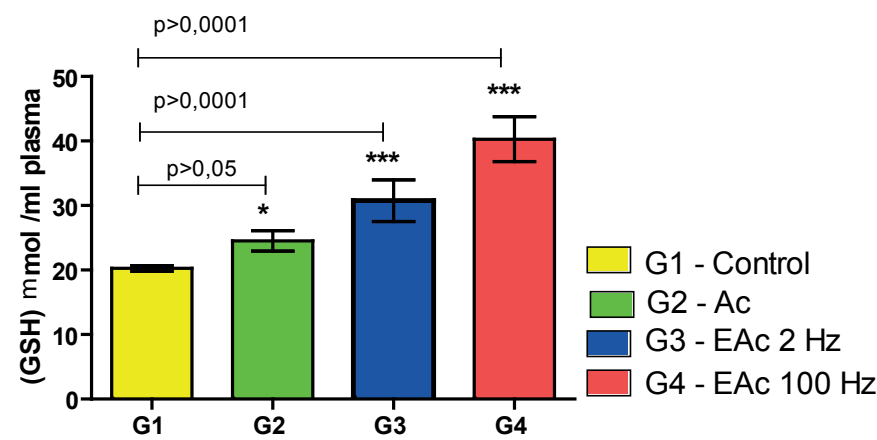

FIGURE 2 - Plasma glutathione (GSH) levels on day 10 of the experiment. Bars represent mean $\pm \mathrm{SD}$ values for each group (G1 - Control, G2 Acupuncture, G3 - Electroacupuncture 2Hz and G4 - Electroacupuncture $100 \mathrm{~Hz}$ ). GSH expressed as microMol $/ \mathrm{ml}$ of plasma. ANOVA/Dunnett multiple comparison tests. ${ }^{* * *} p<0.0001,{ }^{*} p<0.05$ compared with control.
Tissue (ovary) GSH concentrations increased significantly in rats submitted to EA $2 \mathrm{~Hz}$ and EA $100 \mathrm{~Hz}(\mathrm{p}<0.001)$, compared with control (Figure 3).

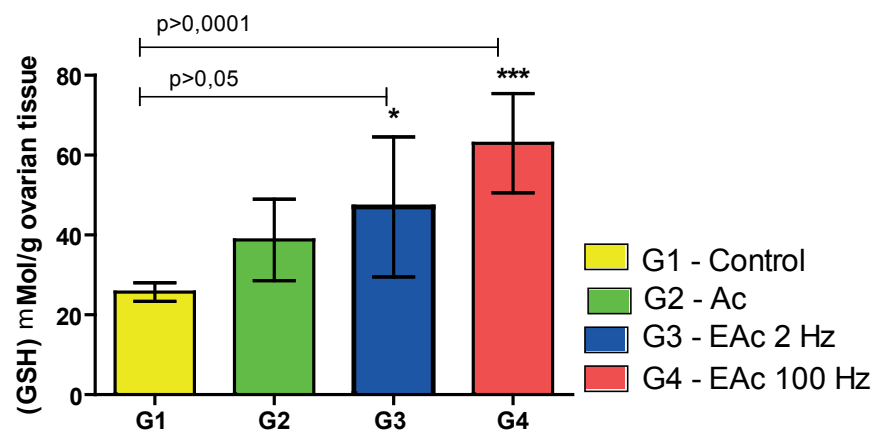

FIGURE 3 - Ovary glutathione (GSH) levels on day 10 of the experiment. Bars represent mean $\pm \mathrm{SD}$ values for each group (G1 - Control, G2 Acupuncture, G3 - Electroacupuncture $2 \mathrm{~Hz}$ and G4 - Electroacupuncture $100 \mathrm{~Hz})$. GSH expressed as microMol/g of ovarian tissue. ANOVA/ Dunnett multiple comparison tests. ${ }^{* * *} \mathrm{p}<0.0001,{ }^{*} \mathrm{p}<0.05$ compared with control.

\section{MDA assay}

Plasma MDA concentrations increased significantly in rats submitted to EA $2 \mathrm{~Hz}(\mathrm{p}<0.01)$ and $\mathrm{EA} 100 \mathrm{~Hz}(\mathrm{p}<0.001)$, compared with control group (Figure 4).

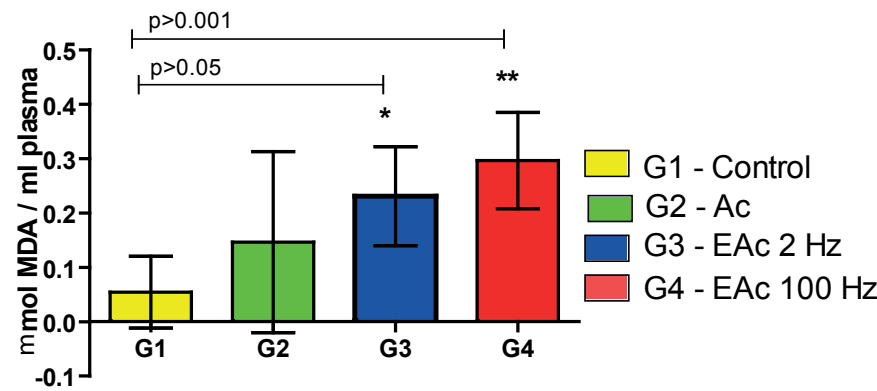

FIGURE 4 - Plasma malonaldehyde (MDA) levels on day 10 of the experiment. Bars represent mean $\pm \mathrm{SD}$ values for each group (G1 Control, G2 - Acupuncture, G3 - Electroacupuncture 2Hz and G4 Electroacupuncture $100 \mathrm{~Hz}$ ). MDA expressed as microMol $/ \mathrm{ml}$ of plasma. ANOVA/Dunnett multiple comparison tests. $* * p<0.001, \quad{ }^{*} \mathrm{p}<0.05$ compared with control.

Ovary MDA concentrations increased significantly in rats submitted to EA $2 \mathrm{~Hz}(\mathrm{p}<0.01)$ and EA100Hz $(\mathrm{p}<0.001)$, compared with control group (Figure 5). 


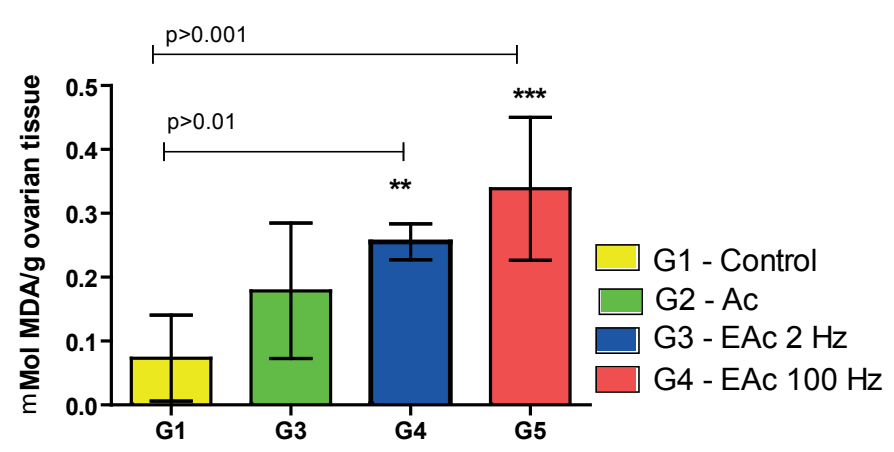

FIGURE 5 - Tissue (ovary) malonaldehyde (MDA) levels on day 10 of the experiment. Bars represent mean $\pm \mathrm{SD}$ values for each group (G1 - Control, G2 - Acupuncture, G3 - Electroacupuncture 2Hz and G4 Electroacupuncture $100 \mathrm{~Hz}$ ). MDA expressed as microMol/g of plasma. ANOVA/Dunnett multiple comparison tests. ${ }^{* * *} \mathrm{p}<0.0001,{ }^{* *} \mathrm{p}<0.01$ compared with control.

\section{MPO activity}

MPO activity decreased significantly in all groups compared with G1 (Figure 6).

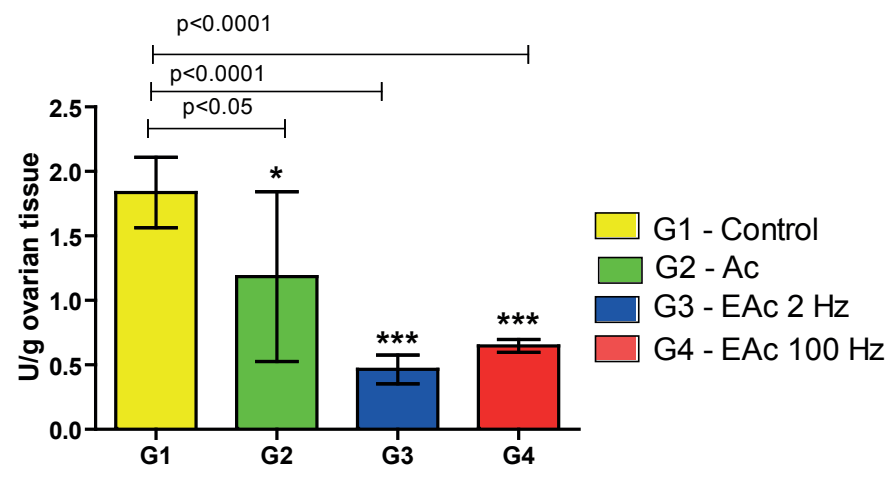

FIGURE 6 - Tissue (ovary) myeloperoxidase (MPO) activity levels on day 10 of the experiment. Bars represent mean \pm SD values for each group (G1 - Control, G2 - Acupuncture, G3 - Electroacupuncture 2Hz and G4 - Electroacupuncture $100 \mathrm{~Hz}$ ). MDA expressed as microMol/g of plasma. ANOVA/Dunnett multiple comparison tests. $* * * p<0.0001, * p<0.05$ compared with control.

\section{Discussion}

Sex steroids play a very important role both in the female reproductive system as in the male, through its regulatory effect in the various sexual and reproductive endocrine functions. These steroid hormones, particularly estrogen, are important for the development of the sexual phenotype, morphology of the reproductive tract and sexual differentiation of the central nervous system. In mammals, sex steroids play a key role in regulating the hypothalamic-pituitary-ovarian, ovarian folliculogenesis, ovulation and embryo implantation ${ }^{22}$. Many studies have indicated that dexamethasone induces free radical toxicity in humans and animals $^{9-11}$. Recent experimental studies have demonstrated that chronic estradiol exposure induces oxidative stress in the hypothalamus of rats ${ }^{13}$.

PCOS has been experimentally induced with a single dose of EV (4 mg) applied to virgin adult cycling SpragueDawley rats ${ }^{14}$. In another study ${ }^{15} 8$-week-old rats receiving an i.m. injection of EV $4 \mathrm{mg}$ developed PCOS. Sasikala et al. ${ }^{23}$ treated female rats with letrozole (an inhibitor of the aromatase enzyme, responsible for the conversion of androgens into estrogens) for 28 days, resulting in alterations compatible with PCOS Surprisingly, healthy rats subjected to a single high dose EV presented no significant alterations in ovarian structure in this study. The explanation for the absence of the expected development of PCOS in female rats exposed to EV, using the same doses as other authors $^{14-15}$ is not clear.

The equivalent of the human right ST-36 (Zusanli) and SP-6 (Sanyinjiao) acupoints were chosen for needling and electrical stimulation. The Zusanli and Sanyinjiao acupoints are located on the femoral quadriceps muscle (in the belly of the anterior tibialis muscle) and on the digitorum profundus flexor muscle, respectively, innervated by branches of the emerging nerve roots from L4 to S1, and is related to somatic and autonomic innervation of pelvic segments, responsible for the regulation of its various neuroendocrine and sensory functions ${ }^{24}$. Ai-Hui et $a l{ }^{25}$ mapped the territories of $\mathrm{C}$ fibers of the superficial peroneal, sural and tibial saphenous nerves in rats, and verified that the rats hind legs are anatomically identical to the lower limbs of human beings, thus justifying the identification of the acupuncture points in the rodent, which would occupy position and action similar to points in humans.

Yu et al. ${ }^{26}$ evaluated the role of needle stimulation of four different acupoints: GB-34 (Yanglingquan), LR-3 (Taichong), ST36 (Zusanli) and SP-10 (Xuehai) acupoints on regulating oxidative stress in the nigrostriatal system in the 6-hydroxydopamine lesioned rat and concluded that acupuncture stimulation prevented the reduction of GSH level as well as the increase in MDA level. In our study, the stimulation of two acupoints, ST-36 (Zusanli) and SP-6 (Sanyinjiao), using manual Ac and EAc with low (2 Hz) and high $(100 \mathrm{~Hz})$ frequencies promoted a significant increase in systemic (plasma) and local (ovary) GSH levels compared with G1 levels. The use of EAc has promoted an increase in GSH levels in the liver and kidneys and in random skin flaps of rats, as demonstrated in previous studies ${ }^{16-17}$. Lipid peroxidation was more intense in rats treated with EAc as there was a significant raise in MDA levels in G3 and G4 rats. However, the use of Ac did not 
alter MDA levels. Despite the fact that is known that there is a correlation observed between the plasma levels of MDA, and the level of 17-beta estradiol where high levels of this hormone may lead to oxidative stress ${ }^{27}$ we cannot exclude the effects of EAc on the increase in lipid peroxidation. We have reported similar results in other tissues in two previous studies ${ }^{16-17}$.

In this study MPO activity decreased significantly in all groups compared with G1, after five sessions of AC and EAC. Recently we have reported similar results in random skin flaps treated with $\mathrm{EAc}^{17}$.

Furthermore, increased levels of MDA in rats treated with EAc shows that the electric stimulation of accupoints may have a pro-peroxidative effect. Both Ac and EAc reduced MPO activity in ovarian tissue, possibly by inhibiting neutrophil infiltration, suggesting that these methods of treatment may have a protective role in the estradiol -induced oxidative injury.

\section{Conclusion}

As evidenced by the changes in glutathione and malonaldehyde levels and myeloperoxidase activity, the results of the present study demonstrate that both acupuncture and electroacupuncture treatments attenuates systemic and local estradiol -induced oxidative damage.

\section{References}

1. Halliwell B, Gutteridge JM. Lipid peroxidation, oxygen radicals, cell damage, and antioxidant therapy. Lancet. 1984;1:1396-7.

2. Turrens JF. Mitochondrial formation of reactive oxygen species. J Physiol. 2003;552, 335-44.

3. Behl C. Oestrogen as a neuroprotective hormone. Nat Rev Neurosci. 2002;3:433-42.

4. Jauslin ML, Meier T, Smith RA, Murphy MP. Mitochondria-targeted antioxidants protect Friedreich Ataxia fibroblasts from endogenous oxidative stress more effectively than untargeted antioxidants. FASEB J. 2003; 17:1972-4.

5. Lodi A, Tonon C, Calabrese V, Schapira AHV. Friedreich's Ataxia: from disease mechanisms to therapeutic interventions. Antioxid Redox Signal 2006;8:438-43.

6. Turgay M, Turgay F, Devrim E, Kucuksahin O, Caydere M, Durak I. The effects of dexamethasone on oxidant/antioxidant status in kidneys of rats administered mercuric chloride. Bratisl Lek Listy. 2012;113(1):10-3.

7. Persky AM, Green PS, Stubley L, Howell CO, Zaulyanov L, Brazeau GA, Simpkins JW. Protective effect of estrogens against oxidative damage to heart and skeletal muscle in vivo and in vitro. Proc Soc Exp Biol Med. 2000;223(1):59-66.

8. Hamden K, Carreau S, Ellouz F, Masmoudi H, El FA. Protective effect of 17beta-estradiol on oxidative stress and liver dysfunction in aged male rats. J Physiol Biochem. 2007;63(3):195-201.

9. Analía MF, Ramiro F, Nicolás F, Juan IR, Roberto C, Daniel, PC. Neuroprotective effect of melatonin on glucocorticoid toxicity in the rat hippocampus. Open Physiol J. 2008;1:23-7.

10. Inkielewicz-Stepniak I, Czarnowski W. Oxidative stress parameters in rats exposed to fluoride and caffeine. Food Chem Toxicol. 2010;48:1607-11.

11. You JM, Yun SJ, Nam KN, Kang C, Won R, Lee EH. Mechanism of glucocorticoid-induced oxidative stress in rat hippocampal slice cultures. Can J Physiol Pharmacol. 2009;87:440-7.

12. Thilagam H, Gopalakrishnan S, Qu HD, Bo J, Wang KJ. $17 \beta$ estradiol induced ROS generation, DNA damage and enzymatic responses in the hepatic tissue of Japanese sea bass. Ecotoxicology. 2010;19(7):1258-67.

13. MohanKumar SMJ, Kasturi BS, Shin AC, Balasubramanian P, Gilbreath ET, Madhan Subramanian M, MohanKumar PS. Chronic estradiol exposure induces oxidative stress in the hypothalamus to decrease hypothalamic dopamine and cause hyperprolactinemia. Am J Physiol Regul Integr Comp Physiol. 2011;300(3):R693-9.

14. Stener-Victorin E, Lundeberg T, Waldenström U, Manni L, Aloe L, Gunnarsson S, Janson PO. Effects of electro-acupuncture on nerve growth factor and ovarian morphology in rats with experimentally induced polycystic ovaries. Biol Reprod. 2000;63(5):1497-503.

15. Stener-Victorin E, Ploj K, Larsson BM, Holmäng A. Rats with steroid-induced polycystic ovaries develop hypertension and increased sympathetic nervous system activity. Reprod Biol Endocrinol. 2005;3:44.

16. Lima LP, de Oliveira Albuquerque A, de Lima Silva JJ, Medeiros FD, de Vasconcelos PR, Guimarães SB. Electroacupuncture attenuates oxidative stress in random skin flaps in rats. Aesthetic Plast Surg. 2012;36(5):1230-5.

17. Silva AH, Figueiredo LM, Dias PA, Prado Neto AX, Vasconcelos PR, Guimarães SB. Electroacupuncture attenuates liver and kidney oxidative stress in anesthetized rats. Acta Cir Bras. 2011;26 Suppl 1:60-5.

18. World Health Organization (WHO), 1993: Standard Acupuncture Nomenclature: a brief explanation of 361 classical acupuncture points and their multilingual comparative list. 2ed. WHO Regional Office for the Western Pacific; 1993.

19. Yin CS, Jeong HS, Park HJ, Baik Y, Yoon MH, Choi CB, Koh HG. A proposed transpositional acupoint system in a mouse and rat model. Res Vet Sci. 2008;84(2):159-65.

20. Uchiyama M, Mihara M. Determination of malondialdehyde precursor in tissues by thiobarbituric acid test. Anal Biochem. 1978(1);86:271-8

21. Sedlak J, Lindsay RH. Estimation of total, protein-bound, and nonprotein sulfhydryl groups in tissue with Ellman's reagent. Anal Biochem. 1968;25(1):192-205.

22. Shirwalkar H, Modi D, Maitra A. Exposure of adult rats to estradiol valerate induces ovarian cyst with early senescence of follicles. Mol Cell Endocrinol. 2007;272(1-2):22-37.

23. Sasikala SL, Shamila S, Nagara Jan, S, Nisha JC, Geetha P, Kishorra JSA. Comparative study of ashokarishtam and clomiphene citrate in combating polycystic ovary syndrome induced oxidative stress in rat. J Cell Tissue Res. 2010;10(1):2105-8.

24. Martins EI, Garcia EG. Pontos de acupuntura: guia ilustrado de referência. São Paulo: Editora Roca; 2003.

25. Ai-Hui LL, Jun-Ming Z, Yi-Kuan X. Human acupuncture points mapped in rats are associated with excitable muscle/skin-nerve complexes with enriched nerve endings. Brain Res. 2005;1012:1549.

26. Yu YP, Ju WP, Li ZG, Wang DZ, Wang YC, Xie AM. Acupuncture inhibits oxidative stress and rotational behavior in 6-hydroxydopamine lesioned rat. Brain Res. 2010;1336:58-65.

27. Sugino N, Karube-Harada A, Taketani T, Sakata A, Nakamura Y. 
Withdrawal of ovarian steroids stimulates prostaglandin F2alpha production through nuclear factor-kappaB activation via oxygen radicals in human endometrial stromal cells: potential relevance to menstruation. J Reprod Dev. 2004;50(2):215-25.

\section{Correspondence:}

Sergio Botelho Guimarães

Rua Professor Costa Mendes, $1608 / 3^{\circ}$ andar

60430-140 Fortaleza-CE Brasil

Tel.: $(55$ 85)3366-8083

Fax: (55-85)3366-8064

sergiobotelho@terra.com.br

Received: April 10, 2013

Review: June 12, 2013

Accepted: July 16, 2013

Conflict of interest: none

Financial source: none

${ }^{1}$ Research performed at Experimental Surgery Laboratory (LABCEX), Faculty of Medicine, Federal University of Ceara (UFC), Fortaleza-CE, Brazil. Part of Master degree thesis, Postgraduate Program in Surgery. Tutor: Prof. Sergio Botelho Guimarães. 